\title{
DYNAMICS OF MOLECULES IN A SUPERCOOLED WATER NANOPARTICLE DURING THE ICE ACCRETION ON THE AIRCRAFT SURFACE
}

\author{
I. A. Amelyushkin ${ }^{1}$ and A. L. Stasenko ${ }^{1,2}$ \\ ${ }^{1}$ Central Aerohydrodynamic Institute (TsAGI) \\ 1, Zhukovsky Str., Zhukovsky, Moscow Region 140180, Russia \\ ${ }^{2}$ Moscow Institute of Physics and Technology \\ Dolgoprudny, Moscow Region 141700, Russia
}

\begin{abstract}
The principal aim of this work is to elaborate a robust physical model and the corresponding numerical code for prediction of the icing startup due to numerous water nanoparticles in the supercooled humid air. For this purpose, a scientified approach was used which is based not on the quantum-mechanics considerations but on the information about intermolecular potentials (especially, Lennard-Jones (LJ), etc.) tightly connected with the state equations of the corresponding specie (e.g., van der Waals for air and water and Mie-Grünaisen for circumfluent body). In other words, the principal idea of this work is to adequately ascribe certain macroscopic characteristics of a water nanoparticle which may significantly differ from those indicated in physical reference books for bulk materials.
\end{abstract}

\section{INTRODUCTION}

The process of ice accretion on a body surface is being under investigation during almost the whole aviation history but up today, the physics of this process needs more deep understanding. It concerns, especially, an initial touch of a supercooled drop to a solid-body surface. Experimental and numerical simulation of ice accretion of bodies in the air flow with drops has been carried out for more than 80 years (see, e.g., [1-4]). Physical processes which take place before water particles approach the surfaces of elements of an aircraft construction (which determines a wetting region) as well as accreted ice evolution were investigated in $[5,6]$. Supercoolled water droplet crystallization after impingement and its adhesion (to an aircraft surface) mechanisms are the fundamental problems in mechanics of multiphase flows as well as in a condensed-matter physics.

This is an Open Access article distributed under the terms of the Creative Commons Attribution License 4.0, which permits unrestricted use, distribution, and reproduction in any medium, provided the original work is properly cited. 
At all temperatures, gas consists not only of molecules (monomers) but also of molecular clusters - small nanoparticles with specific properties and laws of behavior. In particular, their presence makes unnecessary to achieve a critical nucleus $a_{c}^{*}$ of infinitely large radius (in a moment of vapor saturation), which follows from a well-known Thomson's formula:

$$
a_{c}^{*}=\frac{2 \sigma}{\rho_{l} R_{v} T} \ln \frac{p_{v}}{p_{\mathrm{sv}}} .
$$

This formula also gives an infinity value of $a_{c}^{*}$ for saturation pressure $\left(p_{v}\right.$ $\left.=p_{\mathrm{sv}}(T)\right)$. In (1), $\sigma$ and $\rho_{l}$ are the water surface tension coefficient and liquid density, respectively; and $R$ is the universal gas constant. Consider a nanoparticle to be a molecular cluster which consists of more than $N=100$ molecules. That is to say, it is impossible to use only quantum chemistry ab initio calculations in order to define nanodroplet behavior both in time and space. In most cases, it is impossible to define an aggregate state of this particle: some of its molecules behave like gas molecules, others like the liquid ones, and the third ones have kinetic energy which corresponds to temperatures which are close to absolute zero temperature. After impingement on a cold aircraft surface, water nanoparticles may form a water film with a width of several water molecule diameters which will make for an intensive ice accretion regardless of physicochemical properties of an aircraft surface. In the present paper, a model of nanoparticle interaction with a solid body allows to determine condition at which a nanoparticle could freeze down to a body surface.

As the characteristic scale (either spatial or temporal) of fluids is getting smaller, investigations with experimental apparatus become difficult. Conventional theoretical/numerical approaches can also be problematic when continuum mechanics and kinetic theory of fluid are no longer assumed a priori. A use of quantum chemistry methods [7,8] based on an approximate solution of a Shrödinger equation leads to ambiguous solutions and is extremely problematic for large molecular clusters $(N \gtrsim 20)$. Sometimes, theoretical approaches (e.g., the well-known Young-Laplace equation for mechanical balance) lead to incredible conclusions and unsettled problems, while experimental investigation of nanoscale particles require extremely sophisticated techniques.

Another approach for nanoscale fluid phenomena is used in this work. Interaction of water nanoparticles in a carrying air with solid surface of the body is investigated via molecular dynamics (MD) simulation techniques. This approach allows to calculate macroscopic characteristics (such as mass density, pressure, surface tension, etc.) which depend on $N$ and tend to table values for a particle material when $N \rightarrow \infty$. In comparison with the similar investigations (see, e.g., [9-12]), in this paper, the more elaborated model is presented which takes into account some essential physical effects and additional parameters that are controlled during the water particle approaching and impingement (on the aircraft surface) investigation via MD technique. 


\section{PHYSICAL MODEL, SIMULATION CONDITIONS}

Throughout the simulation works in this paper, an MD technique was adopted and based on the classical Newtonian mechanics. A model with the well-known symmetrical LJ two-body interaction for the particle diameter was used to investigate time- and volume-averaged fluid properties. When necessary, more elaborated interaction models and techniques (e.g., more complex intermolecular interaction potentials to investigate water crystals formations and physical mechanisms of self-organized systems [13], nonsymmetrical potentials with charged and ionized molecules $[12,14,15]$, quantum correction generalization water molecular dynamics [16], etc.) could be utilized. Potential energy of pair interaction $U(r)$ can be represented as a sum of orientation, induction, dipole interaction energy, and also a repulsion interaction energy:

$$
U(r)=U_{\text {or }}(r)+U_{\text {ind }}(r)+U_{\text {disp }}(r)+U_{\text {rep }}(r)
$$

where

$$
\begin{aligned}
& U_{\mathrm{or}}(r)=-\frac{p_{e}^{4}}{24 \pi^{2} \varepsilon_{0}^{2} k_{\mathrm{B}} T} \frac{1}{r^{6}} ; U_{\mathrm{ind}}(r)=-\frac{\alpha p_{e}^{2}}{8 \pi^{2} \varepsilon_{0}^{2}} \frac{1}{r^{6}} ; \\
& U_{\text {disp }}(r)=-\frac{3}{4} \alpha^{2} E_{I} \frac{1}{r^{6}} ; \quad U_{\text {rep }}(r) \sim \frac{1}{r^{12}} .
\end{aligned}
$$

Here, $p_{e}$ is the dipole momentum of a molecule; $\alpha$ is the molecular polarizability; $E_{I}$ is the energy of ionization; $\varepsilon_{0}, k_{\mathrm{B}}$, and $\hbar$ are the electrical, Boltzmann, and Plank constants, respectively; and $r$ is the distance between centers of inertia of two molecules. A repulsion energy corresponds to the well-known quantum mechanical Pauli exclusion principle that no two identical fermions (particles with half-integer spin) may occupy the same quantum state simultaneously. A system of molecular dynamic equations of the $i$ th water molecule with a mass $m_{\mathrm{H}_{2} \mathrm{O}}$ is as follows:

$$
\begin{aligned}
m_{\mathrm{H}_{2} \mathrm{O}} \frac{d V^{i}}{d t}=\sum_{j=1}^{N_{\mathrm{H}_{2} \mathrm{O}}\left\{-\nabla U_{\mathrm{H}_{2} \mathrm{O}}^{i j}\right\}} & +\sum_{k=1}^{N_{\text {air }}}\left\{-\nabla U_{\mathrm{H}_{2} \mathrm{O}-\text { air }}^{i k}\right\} \\
& +\sum_{m=1}^{N_{\text {wall }}}\left\{-\nabla U_{\mathrm{H}_{2} \mathrm{O}-\text { wall }}^{i m}\right\}+m_{\mathrm{H}_{2} \mathrm{O}} g+F_{\text {dis }} .
\end{aligned}
$$

The first, the second, and the third components of Eq. (3) are the forces which act on the $i$ th molecules from other water molecules, from air molecules, and from the molecules of the surface, respectively. The last component, $F_{\text {dis }}$, describes the energy dissipation of the molecule which could exist. In this work, it was not taken into account. The force of gravity is neglected. The LJ potential parameters were taken from the well-known Van der Waals equation of 
state constants: $\sigma_{\mathrm{H}_{2} \mathrm{O}}=2.6 \AA$ and $\varepsilon_{\mathrm{H}_{2} \mathrm{O}}=809 k_{\mathrm{B}} \cong 1.12 \cdot 10^{-20} \mathrm{~J}$. The wall temperature was defined via thermal quantum oscillation of the atoms of the aircraft's surface crystal lattice. It was possible to estimate characteristic frequencies and amplitudes of atoms of nanostructured hydrophobic material for the aircraft surface.

A liquid particle consisting of 50-15000 molecules was generated and equilibrated at various temperatures close to the temperature of a flying vehicle in the aerosol flow. The space around a particle is a cubic region; symmetrical boundary conditions for molecules were set for all horizontal and top directions. The characteristic size of a cubic region is by one-two orders higher than the characteristic size of a nanoparticle. On the bottom of a cubic cell, a wall boundary condition was set. Assuming a potential interaction among particles (atoms or molecules), the equations of motion are numerically integrated to trace the particle motions according to the algorithms which are close to the ones described in $[14,17]$. The Botlzman-Maxwell velocity distribution function was used for molecules velocities in order to define a drop temperature. Onto the bottom wall, the water nanoparticle was impinged at various angles with various mass center initial speeds $V_{\mathrm{imp}}$ of nanoparticle from a fixed position. During the simulation, water molecular radial distribution of velocity distribution function, density, temperature, intermolecular energy density, and pressure were controlled. For sure, these parameters should depend on the dimension of the molecular cluster a nanoparticle. For example, the well-known Tolman's formula [18] for dependence of surface tension $\sigma_{l}$ on a drop radius may be regarded as an attempt to reveal such a dependence. Intermolecular frequencies and amplitudes were calculated according to ab initio quantum chemistry calculations. The temperature was controlled as an average thermal kinetic energy of molecules in a reference frame connected with the inertial center of a nanoparticle. Calculating the speed of the inertial center of a nanoparticle, it was assumed that a molecule belongs to a particle if this molecule is located in the area with a particulate density more than $10 \mathrm{~kg} / \mathrm{m}^{3}$.

\section{PRESENTATION OF MACROPARAMETERS IN TERMS OF NANOPARAMETERS}

\subsection{Temperature and Velocity Distribution of Molecules}

Molecules were given velocities according to a well-known Maxwell-Boltzmann molecule velocity distribution law in a reference frame, which is connected with the mass center of a nanoparticle. During all the simulation, the molecule velocity distribution was controlled as well as the temperature. Numerical simulation showed an appearance of thermal oscillations which were gradually reducing with 


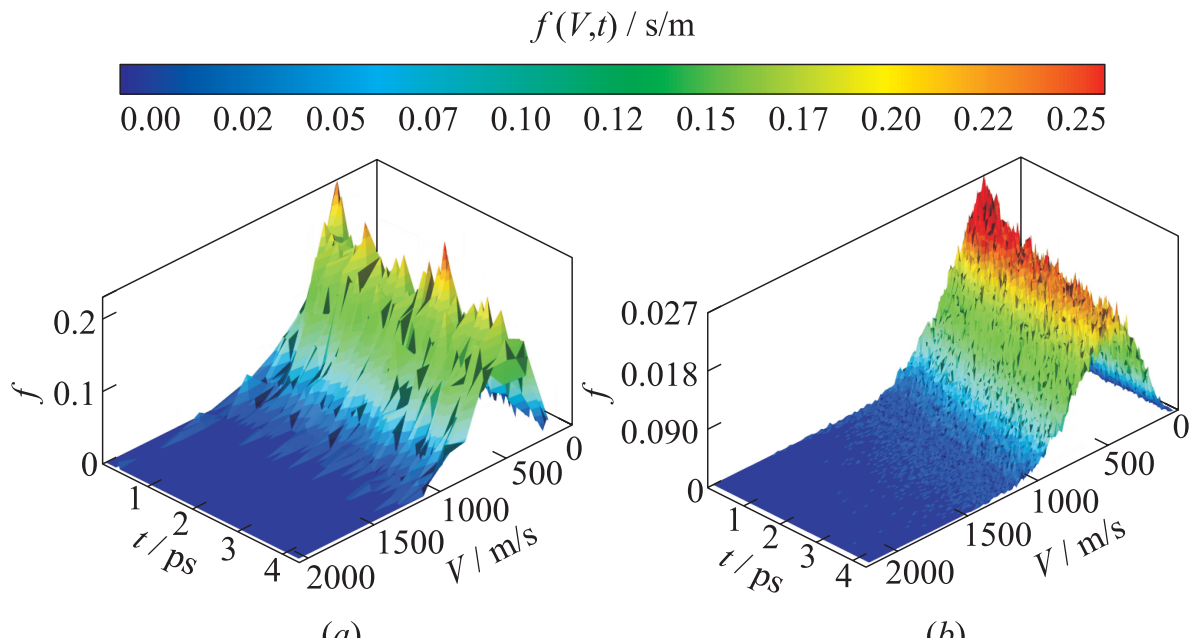

(a)

(b)

Figure 1 Molecular velocity distribution function in time for tiny water cluster $(a)$ ( $\sim 160$ molecules $)$ and for droplet $(b)(\sim 2500$ molecules $)$

time. During a numerical molecular dynamic simulation of a particle, the fastest molecules near and under its surface can leave the drop and, as a result, the molecular velocity distribution function changes. Some of water vapor molecules impact with a particle and, as a result, they may become a part of this particle changing its temperature and molecular velocity distribution function. Finally, a dynamic equilibrium appears and amplitudes of oscillations reduce with an increase of a number of molecules (Fig. 1). Numerical experiment showed that inside a nanoparticle, a kinetic energy of molecules as well as a temperature is lower than on the interphase drop boundary. This effect reduces with the increase of a drop size. It should be noted that as all molecules in a particle have significantly different velocities the particle temperature distribution is also nonuniform. Temperature fluctuations in time and space were approximately $10{ }^{\circ} \mathrm{C}$.

\subsection{Density Calculation}

Density $\rho$ of a nonoparticle depends on its size. With the increasing of the particulate size $R_{p}$, its density tends to be closer to its well-known value in the reference books. It should be noted that a thickness of interphase boundary of a nanoparticle is comparable with a particulate size itself. Thermal motion and oscillation of molecules lead to density fluctuations. In order to calculate a radial density distribution $\rho(r)$ ( $r$ is the distance from drop's mass center) in a particle 


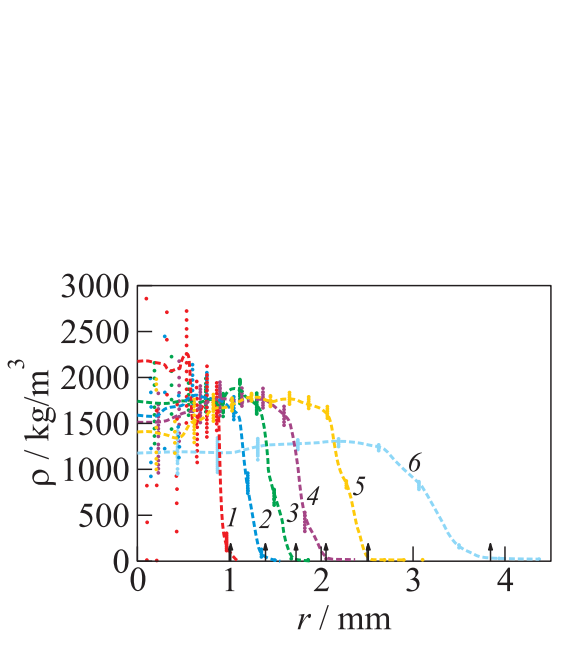

(a)

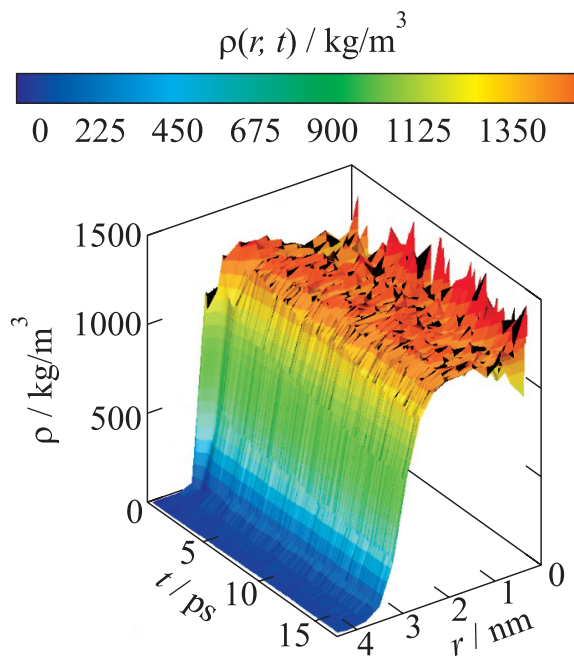

(b)

Figure 2 Drop density radial distribution depending on water particle size $(a)$ and density radial and temporary distribution for a droplet of 1080 molecules $(b)$. Temperature is $10{ }^{\circ} \mathrm{C}$

let split its volume into spherical layers. In each layer, the number of molecules which belong to these layers divide on a layer's volyme $\Delta V_{d}$ and multiply by the mass of a single molecule $m_{\mathrm{H}_{2} \mathrm{O}}$ :

$$
\rho(r)=m_{\mathrm{H}_{2} \mathrm{O}} \frac{N(r+\Delta r)-N(r)}{\Delta V_{p}}
$$

where

$$
\Delta V_{p}=\frac{4}{3} \pi(r+\Delta r)^{3}-\frac{4}{3} \pi r^{3} .
$$

Figure 2 illustrates the dependence of a particle's density radial distribution on a water particle size at a temperature of surrounding wet (here, the humidity of the air is approximately $5 \%$ ) air approximately $10{ }^{\circ} \mathrm{C}$. Curve 1 corresponds to a particle which consists of about 160 molecules. Curve 6 corresponds to a particle which contains approximately 4660 molecules. This figure shows that the particulate density becomes larger as the particle size becomes smaller. Density fluctuations increase with the diminishing of a particle size. It is due to increasing of contribution of single molecules into calculated density. When a particle size significantly larger than a characteristic size of a molecule, particle's density tends to be closer to its well-known table value in the reference books. By 


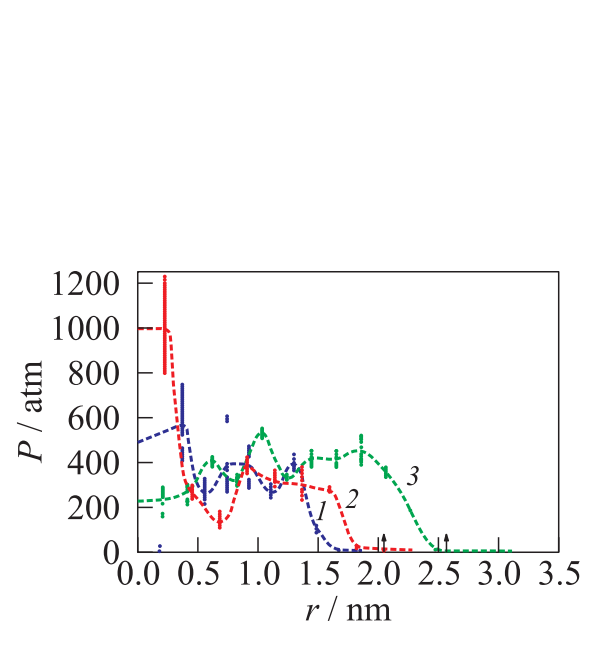

(a)

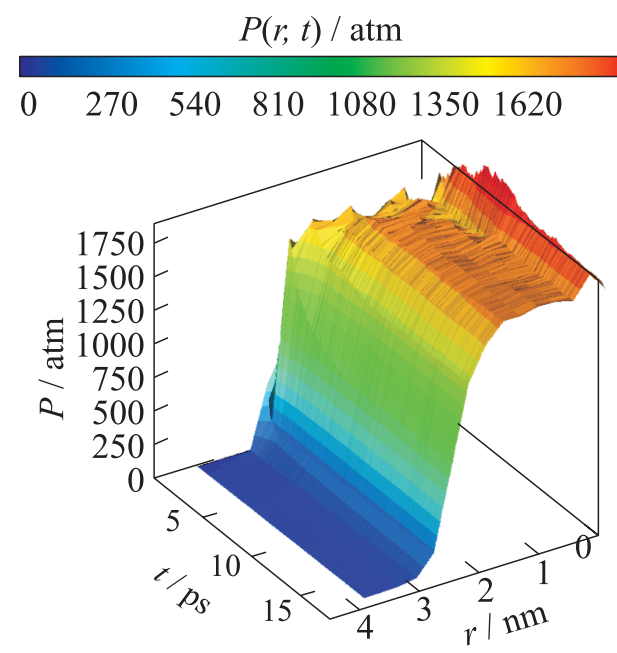

(b)

Figure 3 Pressure in a water particle radial distribution depending on a particle size $(a)$ and pressure in a water particle radial and temporary distribution for a particle of 1080 molecules $(b)$. Temperature is $\approx 10{ }^{\circ} \mathrm{C}$

the way, let estimate the density of a water particle which consists from one molecule:

$$
\rho\left(\sigma_{\mathrm{H}_{2} \mathrm{O}} / 2\right)=\frac{m_{\mathrm{H}_{2} \mathrm{O}}}{(4 / 3) \pi\left(\sigma_{\mathrm{H}_{2} \mathrm{O}} / 2\right)^{3}} \approx 3250 \mathrm{~kg} / \mathrm{m}^{3} .
$$

It is three times bigger than a liquid water density at normal conditions. It is interesting to note that a mass density in a particle is higher near the interphase boundary and strongly depends on a radius of curvature of a particle. Figure 3 also shows a smooth diminishing of a particulate density with the increase of a distance from the interphase "boundary." The larger is the particle, the thicker is the interphase boundary and the smaller is the density peak in the interphase "boundary." So, a particulate density distribution depends on temperature, amount and humidity of surrounding air, and the character of interaction of its molecules.

Figure 2 shows that increasing of water particle size leads to the "normal" density $1000 \mathrm{~kg} / \mathrm{m}^{3}$. This figure also shows that the water radial density thermal fluctuations vanish with the increase of a particle size. A drop density in the atmosphere was also experimentally found to be 2-2.5 larger than water density at normal conditions [19]. According to classical concepts, an interphase boundary has a transient layer liquid-vapor: a density gradually decreases on this 
"boundary." On a "boundary" itself, there is a certain density drop of a finite size; with an increasing distance from this surface, a vapor density diminishes down to the thermodynamically equilibrium value $\rho_{\mathrm{sv}}(T)$ for saturation conditions for a given temperature [20]. In order to estimate a characteristic thickness $h$ of a transitional layer in a liquid, there is its relation with the surface tension coefficient $\sigma_{l}$ and with the equal radius $\sqrt{\left\langle r_{m}^{2}\right\rangle}$ of action of cohesion forces:

$$
h=\frac{a_{V W}\left\langle r_{m}^{2}\right\rangle}{\sigma_{l}}\left(\rho_{l}-\rho_{\mathrm{sv}}(T)\right)^{2}
$$

where $a_{V W}$ is the parameter of van der Waals state equation. Numerical estimations show that a scale of thickness $h$ is few nanometers. Thus, from a classical point of view, a nanoparticle is a "transitional layer."

\subsection{Pressure Calculation}

Let cover the molecules in a liquid particle by concentric virtual spheres with a radius $r_{k}$ whose centers situate in a mass center of a droplet. Pressure is calculated as a molecules flux of molecules through each sphere. Let $N(t)$ be an amount of water molecules that passes through the $k$ th virtual sphere and produces in a time interval $t$ the following radial kinetic momentum:

$$
\Delta p_{r}^{k}(t)=\sum_{i=1}^{N(t+\Delta t)-N(t)}\left|m_{\mathrm{H}_{2} \mathrm{O}} V_{r}^{i, k}(t) i_{r}\right|=m_{\mathrm{H}_{2} \mathrm{O}} \sum_{i=1}^{N(t+\Delta t)-N(t)}\left|V_{r}^{i, k}(t)\right| .
$$

Here, $V_{r}(r, \theta, \varphi)=v_{x} \sin \theta \cos \varphi+v_{y} \sin \theta \sin \varphi+v_{z} \cos \theta$ is the radial velocity component which depends on polar and azimuth angles. So, pressure radial distribution $P_{k}$ is calculated as a force which acts on a virtual surface with area $4 \pi r_{k}^{2}$ :

$$
\begin{aligned}
P_{k}=P_{k}\left(r_{k}\right)=\frac{\Delta p_{r}^{k}(t)}{4 \pi r_{k}^{2} \Delta t} & =\frac{m_{\mathrm{H}_{2} \mathrm{O}}}{4 \pi r_{k}^{2} \Delta t} \sum_{i=1}^{N(t+\Delta t)-N(t)}\left|V_{r}^{i, k}(t)\right| \\
& =\frac{\varepsilon_{\mathrm{H}_{2} \mathrm{O}}}{\sigma_{\mathrm{H}_{2} \mathrm{O}}^{3}} \frac{1}{4 \pi \bar{r}_{k}^{2} \Delta \bar{t}} \sum_{i=1}^{N(t+\Delta t)-N(t)}\left|\bar{V}_{r}^{i, k}(t)\right|=\frac{\varepsilon_{\mathrm{H}_{2} \mathrm{O}}}{\sigma_{\mathrm{H}_{2} \mathrm{O}}^{3}} \overline{P_{k}}
\end{aligned}
$$

Here, $\varepsilon_{\mathrm{H}_{2} \mathrm{O}} / \sigma_{\mathrm{H}_{2} \mathrm{O}}^{3} \cong 6828$ atm is the pressure that associates pressure with a dimensionless pressure $\overline{P_{k}}$. In Fig. 3, one can see the results of numerical drop's pressure radial distribution: in Fig. $3 a$, there is radial pressure distribution that depends on a drop's size (curves 1, 2, and 3 correspond to droplets which consist of 647, 1080, and 2449 molecules) and in Fig. $3 b$, there is a pressure radial distribution that changes in time and is calculated for a drop which consists of 1080 molecules. 
During the simulations, such macroparameters as radial distribution of temperature and potential energy density of molecular system were investigated in time depending on droplet size, temperature, and the surrounding humid air parameters.

\section{PARTICULATE COAGULATION AND BREAKUP}

In Fig. 4, there is an example of water drops collision in a humid air. Two water particles (of approximately 2500 molecules) move towards one another with equal speed (of their mass centers) $100 \mathrm{~m} / \mathrm{s}$ and have an impact parameter $b_{1} \approx 0.5 \mathrm{~nm}$. After the collision, particles oscillate, rotate, and, finally, split again with other velocities, temperatures, and distance $b_{2}$ between velocities directions. Use of molecular dynamics enables to form a core of a collision integral in the integrodifferential kinetic equation for real gases as well as a molecular mechanism of phase transitions, impact to each other, electrization, and mass-heat exchange rates.

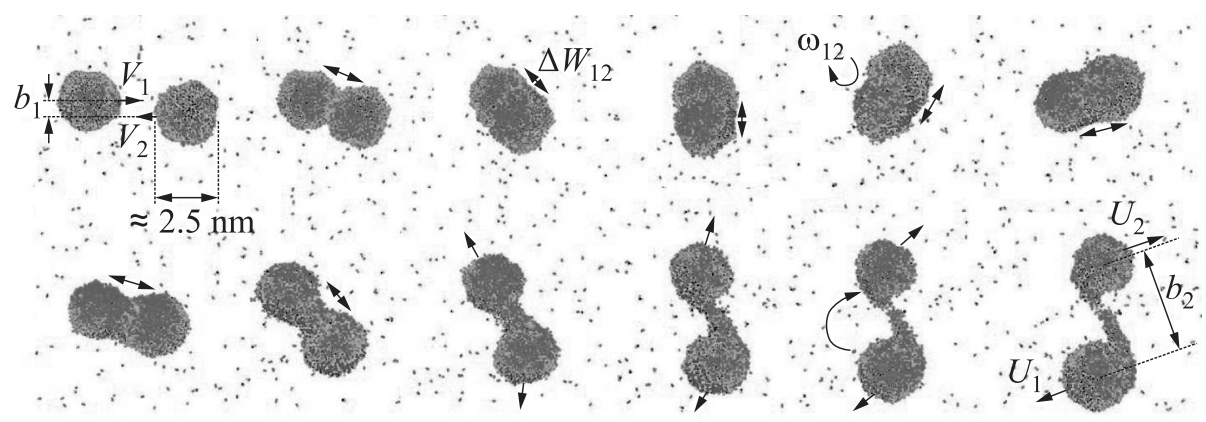

Figure 4 Stages of particles collision, rotation, and breakup

\section{DROPLET APPROACHING AND IMPINGEMENT UPON A "RIGID" BODY}

In order to investigate a particulate behavior (in dependence on its size as on other parameters) in the process of its impingement on the aircraft surface, the physics of this process should be defined. One may calculate amplitudes of atom oscillation of a solid body in the aerosol flow via energy considerations. Energy of phonons of the $i$ th acoustic branch with a wave vector $\mathbf{k}$ is as follows:

$$
E_{i}(\mathbf{k})=\hbar \omega_{i}(\mathbf{k})\left(\left(e^{\hbar \omega_{i}(\mathbf{k}) /\left(k_{\mathrm{B}} T\right)}-1\right)^{-1}+\frac{1}{2}\right)
$$


where $\omega_{i}(\mathbf{k})$ is the frequency of oscillatory mode. From other hand, it is possible to present this energy $E_{i}(\mathbf{k})$ in terms of average potential and kinetic energies of atom's (with a mass $m_{A}$ ) oscillations with an amplitude $\Delta a(\mathbf{k})$. For a harmonic oscillator, an average kinetic energy equals an average potential energy:

$$
E_{i}(\mathbf{k})=2 \frac{m_{A} \omega_{i}^{2}(\mathbf{k}) \Delta a_{i}^{2}(\mathbf{k})}{2}=\hbar \omega_{i}(\mathbf{k})\left(\left(e^{\hbar \omega_{i}(\mathbf{k}) /\left(k_{\mathrm{B}} T\right)}-1\right)^{-1}+\frac{1}{2}\right) .
$$

One can obtain a mean square amplitude of atoms oscillations via the Brillouin zone averaging as well as all the branches of oscillations averaging of $\Delta a_{i}(\mathbf{k})$ :

$$
\Delta a_{i}^{2}(T)=\frac{1}{N_{c}} \sum_{i=1}^{3} \sum_{\mathbf{k}} a_{i}^{2}(\mathbf{k})=\frac{9 \hbar^{2}}{4 m_{A} k_{\mathrm{B}} \theta_{d}} I\left(\frac{T}{\theta_{d}}\right)
$$

where $N_{c}$ is the number of elementary crystal cells and

$$
I\left(\frac{T}{\theta_{d}}\right)=1+4\left(\frac{T}{\theta_{d}}\right)^{2} \int_{0}^{\theta_{d} / T} \frac{x^{2} d x}{e^{x}-1} .
$$

The properties of crystal lattice and the results of numerical calculations are given in Table 1.

For a solid body with a cubic sell crystal lattice, a length of one cell is $a=\sqrt[3]{n \mu /\left(\rho N_{A}\right)} ;$ for ice with a hexagonal cell, $a=\sqrt[3]{2 n \mu_{\text {ice }} /\left(3 \sqrt{3} \rho_{\text {ice }} N_{A}\right)}$.

From the quantum theory, one has:

$$
\frac{k_{w} \Delta a^{2}}{2}=k_{w} \frac{9 \hbar^{2}}{8 k_{\mathrm{B}} \theta_{d}} I\left(\frac{T}{\theta_{d}}\right)=\frac{m_{A}\left\langle c_{w}^{2}\right\rangle}{2}=\frac{k_{\mathrm{B}} T}{2} .
$$

Here, $\left\langle c_{w}^{2}\right\rangle$ is the mean-square velocity of wall atoms.

From the elasticity theory, one has:

$$
k_{w}=\frac{E S}{a}=\frac{E \gamma}{a}\left(\frac{a}{\sqrt[3]{n}}\right)^{2}=\frac{\left(2 k_{\mathrm{B}} \sqrt{\theta_{d} T} / 3 \hbar\right)^{2}}{I\left(T / \theta_{d}\right)}=\frac{J^{2}\left(\theta_{d} T\right)}{I\left(T / \theta_{d}\right)} .
$$

Table 1 Properties of crystal materials at temperature $-10{ }^{\circ} \mathrm{C}$

\begin{tabular}{ccccccccc}
\hline Material & $\rho, \mathrm{kg} / \mathrm{m}^{3}$ & $\mu, \mathrm{g} / \mathrm{mol}$ & $N$ & $a, \AA$ & $E, \mathrm{~Pa}$ & $\theta_{D}, \mathrm{~K}$ & $\Delta a, \AA$ & $\omega, 10^{12} \mathrm{~s}^{-1}$ \\
\hline $\mathrm{Al}$ & 2699 & 26.982 & 4 & 4.049 & $68 \cdot 10^{9}$ & 394 & 0.173 & 16.69 \\
$\mathrm{Fe}$ & 7874 & 55.847 & 2 & 2.867 & $186 \cdot 10^{9}$ & 460 & 0.147 & 19.29 \\
$\mathrm{Ice}$ & 917 & 18.015 & 2 & 2.848 & $5 \cdot 10^{9}$ & 192 & 0.603 & 8.31 \\
\hline
\end{tabular}

Remarks: $\rho-$ density; $\mu-$ molar mass; $N-$ number of atoms per one cell; $a-$ length of one cell; $E-$ Young modulus; $\theta_{D}-$ Debye temperature; $\Delta a-$ atoms amplitude oscillations; and $\omega-$ atoms frequency oscillations. 
Here,

$$
\gamma=\frac{k_{w} a}{E(a / \sqrt[3]{n})^{2}}=\frac{J^{2}\left(\theta_{d} T\right)}{E \sqrt[3]{\mu /\left(n \rho N_{A}\right)} I\left(T / \theta_{d}\right)}
$$

is the coefficient which defines a difference between a theory of elasticity and quantum theory. Calculations show that for wide range of materials, a coefficient $\gamma$ is approximately equals unity $(\gamma \approx 1)$ in a range of temperatures which corresponds to the flying conditions of an aircraft. That is to say, both quantum and elasticity theories can be used in order to estimate solid body atom's oscillations amplitudes and frequencies. From equations

$$
\left\langle(\omega \Delta a)^{2}\right\rangle \cong\left\langle\omega^{2}\right\rangle\left\langle\Delta a^{2}\right\rangle ;\left\langle c_{w}^{2}\right\rangle \cong \frac{k_{\mathrm{B}} T}{m_{A}}
$$

one can obtain

$$
\left\langle\omega^{2}\right\rangle=\frac{J^{2}\left(\theta_{d} T\right)}{I\left(T / \theta_{d}\right)}
$$

There is no need to use molecular dynamics for solid body atoms thermal oscillations simulation, it consumes huge amounts of computer power. In the present work, crystal lattice atoms moved only in a thin layer with a thickness of several atom sizes (Fig. 5). In order to take into account interaction with more "deep" atoms of a solid body (which are below the described above thin layer), let obtain a potential which describes interaction of single water molecule (which is situated in a distance $h$ from a solid body surface) with all atoms of a solid body crystal lattice (see Fig. 5). The wall is assumed to be a crystal lattice whose atoms interact with water molecules via LJ law with the following parameters: $\varepsilon_{w}=\chi \varepsilon_{\mathrm{H}_{2} \mathrm{O}}$ and $\sigma_{w} \approx \sigma_{\mathrm{H}_{2} \mathrm{O}}$. Let split the wall into numerous thin plane layers with a thickness $d y$, all these layers are parallel to a solid body surface (see Fig. 5). Let split each layer on rings with radius $r$ and width $d r$. So, a number of molecules in a layer with the height $d y$ and the area $2 \pi r d r$ equals to $d N$ $=2 \pi r d r d y \rho_{w} / m_{A}$. The potential energy of interaction between a water molecule and a thin ring will be as follows:

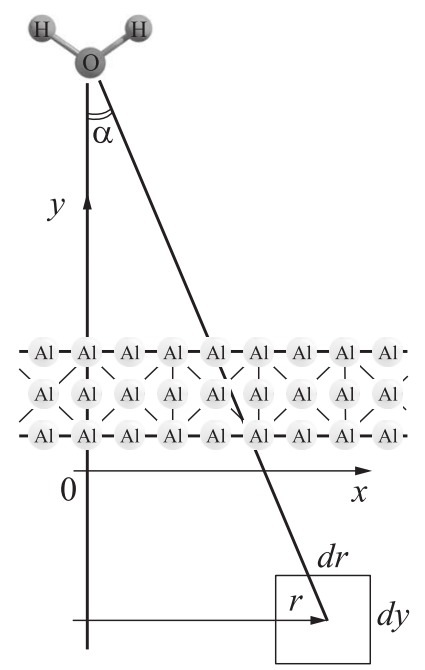

Figure 5 Scheme of interaction of a water molecule with the surface 


$$
\begin{gathered}
d U=d N 4 \varepsilon_{w}\left(\left(\frac{\sigma_{w}}{\sqrt{y^{2}+r^{2}}}\right)^{12}-\left(\frac{\sigma_{w}}{\sqrt{y^{2}+r^{2}}}\right)^{6}\right) \cos \alpha \\
=2 \pi r d r d y \frac{\rho_{w}}{m_{A}} \cdot 4 \varepsilon_{w}\left(\left(\frac{\sigma_{w}}{\sqrt{y^{2}+r^{2}}}\right)^{12}-\left(\frac{\sigma_{w}}{\sqrt{y^{2}+r^{2}}}\right)^{6}\right) \frac{y}{\sqrt{y^{2}+r^{2}}} \\
U(h)=8 \pi \frac{\rho_{w}}{m_{A}} \varepsilon_{w} \int_{y=-\infty}^{h} y d y \int_{r=0}^{\infty}\left(\left(\frac{\sigma_{w}}{\sqrt{y^{2}+r^{2}}}\right)^{12}-\left(\frac{\sigma_{w}}{\sqrt{y^{2}+r^{2}}}\right)^{6}\right) \frac{r d r}{\sqrt{y^{2}+r^{2}}} \\
=\frac{8}{15} \frac{\rho_{w}}{m_{A}} \pi \varepsilon_{w} \sigma_{w}^{3}\left(\frac{5}{33}\left(\frac{\sigma_{w}}{h}\right)^{9}-\left(\frac{\sigma_{w}}{h}\right)^{3}\right)
\end{gathered}
$$

This new potential can be used for calculations of water molecules interaction with a solid body surface. More complex model based on nonsymmetrical potentials $[12,15]$ can be obtained in a similar way.

In Fig. 6, curve 1 corresponds to the ordinary LJ potential interaction between two single molecules $U(r)$. Curve 2 corresponds to the potential interaction between a water molecule and an infinite water body according to Eq. (4). Curves 3 and 4 correspond to the interaction between a water molecule and hydrophilic and hydrophobic surfaces. Finally, curve 5 corresponds to a superhydrofobic surface whose atoms would repulse water molecules at each distance. In this case, a repulsion force between water molecule and a surface is inversely proportional to the distance between a molecule and a surface in the 3rd power.

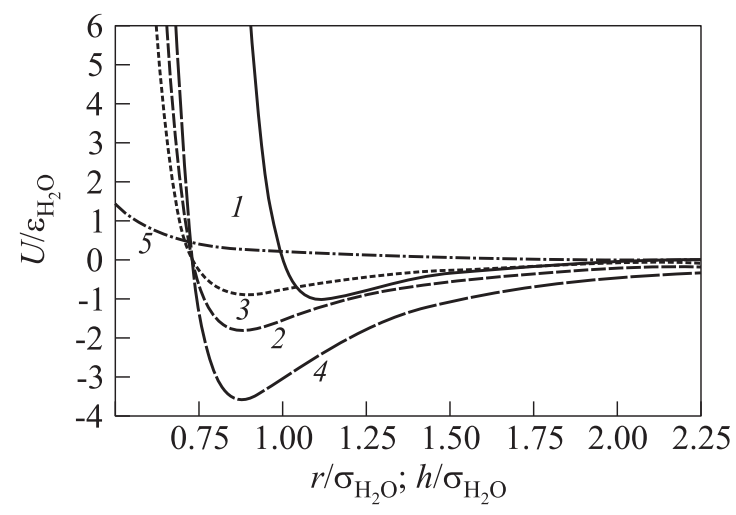

Figure 6 Potentials of molecular interactions: 1 - water LJ potential; 2 - wall potential for water wall; 3 - wall potential of a hydrophobic surface; 4 - wall potential of a hydrophilic surface; and 5 - wall superhydrofobic surface 
Numerical simulations are usually carried out with continuum hydrodynamics schemes, gas kinetic theory equation, and sophisticated quantum-mechanical calculations. Well-known methods of quantum chemistry cause enormous computer power to calculate a molecular configuration of a nanoparticle - a big molecular cluster. Here is a review of the present authors' recent progress in studies of droplet impingement process by MD simulation, in which the influence of governing parameters on a particle dynamics, such as wettability, impingement speed, particulate size, water, air and surface temperature, and energy of surface interaction were investigated. During the impingement, limiting wetting angles in addition to above described physical parameters were estimated and controlled.

In case of hydrophilic surface, a nanoparticle starts to spread after the collision and reaches the final state, in which the shape of nanoparticle depends on the impinging speed. The initial behavior of deformation on a hydrophobic surface looks similar but later, the liquid nanoparticle bounces back.

Various quantities were defined in order to examine the water nanoparticle behavior during the impingement such as particulate height, contact area, contact angles, direction of the liquid flux inside the liquid nanoparticle - the droplet. Figures 7 and 8 are the examples of water particles interactions with hydrophilic and hydrophobic surfaces: one can see that for the same temperatures, the nanoparticle is adhered to a hydrophilic surface and rebounds from a hydrophobic surface.

Hence, these investigations allow to find a region (in a space of a multitudes of aircraft flight parameters: temperature, particulate size, pressure, air density and humidity, etc. and properties of circumfluent body material) in which water particles cannot freeze and adhere to elements of aircraft construction.

There could be two relevant factors [11] in choosing the speed of impingement $V_{\mathrm{imp}}$. One is the Reynolds number $\operatorname{Re}=\rho V_{\mathrm{imp}} R_{p} / \mu_{\mathrm{Air}}$ which indicates the effect of viscosity. The other is the Weber number defined as $\mathrm{We}=\rho V_{\mathrm{imp}}^{2} R_{p} / \sigma_{l}$ that shows how the effective surface tension affects the system. Fluids with the same Re and We are expected to show a similar behavior.

In order to take into account electrization of water drops and particles during their impingement, let consider the following. If a droplet carries charge $q=Z e$ (here, $Z$ is the number of electrons in a drop or a particle), the character and intensity of its interaction with a solid body changes: besides described above (see Eq. (2)), the forces which act on water molecule are the force of molecular theory of electrization:

$$
F_{\text {ewall }}=-\frac{1}{4 \pi \varepsilon_{0}} \frac{e^{2}}{(2 h)^{3}} 2 h .
$$

If the force acting on the electron in a water molecule is higher than a gradient of an electron affinity to a molecule potential $\left(F_{\text {ewall }}>e\left(-\nabla \varphi_{\mathrm{af}}\right)\right)$, this electron begins to move across the water particle towards a solid body. 


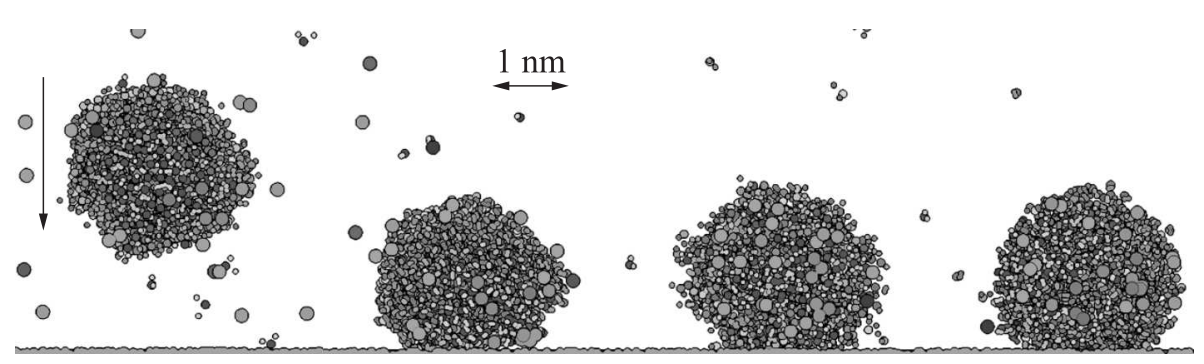

Figure 7 Water nanoparticle wall-mass-heat exchange on a hydrophilic surface; the droplet temperature as well as temperature of surrounding gas and a solid body crystal lattice oscillations is $-10{ }^{\circ} \mathrm{C}$

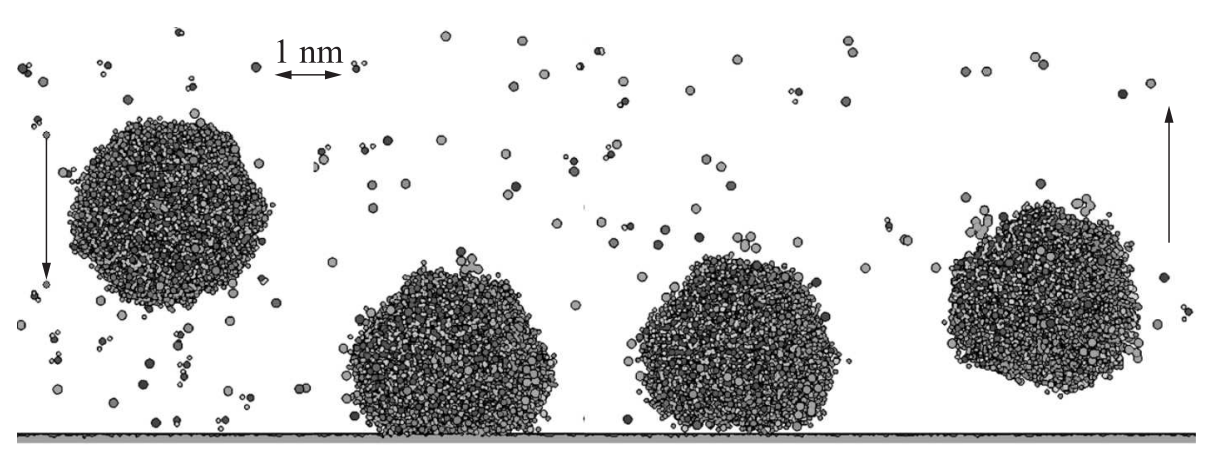

Figure 8 The same as in Fig. 7 but for the droplet two times larger and for hydrophobic surface

\section{EXPERIMENTAL VERIFICATION OF THE RESULTS. AN INVERSE PROBLEM}

To determine the laws of water molecule interaction with solid aircraft surface, let state and solve an inverse problem. According to the physic assumptions, it is worth to consider that ice water molecules interact with crystal lattice atoms via LJ law:

$$
U(r)=4 \varepsilon_{w}\left(\left(\frac{\sigma_{w}}{r}\right)^{12}-\left(\frac{\sigma_{w}}{r}\right)^{6}\right) .
$$

There are two unknown constants $\varepsilon_{w}$ and $\sigma_{w}$ which should be found. If one measures an adhesion force between the ice and the solid body in normal and tangential directions, it is possible to obtain a system of two equations which enables to find the described above constants. 
Let consider a small fragment of a solid body with an area $L \times L$ and a height $H_{\text {wall }}$. The fragment of accreted ice also should be considered with the same horizontal dimensions but a height $H_{\text {ice }}$.

If one summarizes all forces which act between lattice atoms and ice molecules, the adhesion force which acts between ice and a body can be obtained. Knowledge of ice crystal structure as well as a crystal structure of a solid body material enables to set the coordinates of the ice and the solid body. An experimental measurement of force in normal and tangential direction gives two equations from which one can find two constants of the described above potential: $\varepsilon_{w}$ and $\sigma_{w}$. The force which acts between the ice molecule and a solid body atom is as follows:

$$
\begin{aligned}
\mathbf{f}(\mathbf{r})=-\nabla U(\Delta r)=-\frac{\partial U}{\partial r}[ & \left.\frac{\partial r}{\partial x} \mathbf{i}+\frac{\partial r}{\partial y} \mathbf{j}+\frac{\partial r}{\partial z} \mathbf{k}\right] \\
& =\frac{24 \varepsilon_{w}}{\sigma_{w} r^{2}}\left(\left(\frac{\sigma_{w}}{r}\right)^{7}-2\left(\frac{\sigma_{w}}{r}\right)^{13}\right)[x \mathbf{i}+y \mathbf{j}+z \mathbf{k}] .
\end{aligned}
$$

The force which acts between all atoms of an ice crystal and a solid body is as follows:

$$
\mathbf{F}(\mathbf{r})=\sum_{N_{1}} \sum_{N_{2}} \mathbf{f}(\mathbf{r})
$$

or

$$
\begin{array}{r}
\mathbf{F}(\mathbf{r})=\sum_{i_{1}}^{N_{x}^{1}} \sum_{i_{2}}^{N_{x}^{2}} \sum_{j_{1}}^{N_{y}^{1}} \sum_{j_{2}}^{N_{y}^{2}} \sum_{k_{1}}^{N_{z}^{1}} \sum_{k_{2}}^{N_{z}^{2}} \frac{24 \varepsilon_{w}}{\sigma_{w}\left(r_{1}-r_{2}\right)^{2}}\left(\left(\frac{\sigma_{w}}{r_{1}-r_{2}}\right)^{7}-2\left(\frac{\sigma_{w}}{r_{1}-r_{2}}\right)^{13}\right) \\
\times\left[\left(x_{i_{1}}^{1}-x_{i_{2}}^{2}\right) \mathbf{i}+\left(y_{j_{1}}^{1}-y_{j_{2}}^{2}\right) \mathbf{j}+\left(z_{k_{1}}^{1}-z_{k_{2}}^{2}\right) \mathbf{k}\right] .
\end{array}
$$

Here, $r_{1}-r_{2}=\sqrt{\left(x_{i_{1}}^{1}-x_{i_{2}}^{2}\right)^{2}+\left(y_{j_{1}}^{1}-y_{j_{2}}^{2}\right)^{2}+\left(z_{k_{1}}^{1}-z_{k_{2}}^{2}\right)^{2}}$. From Eq. (5), one may have a system of two equations for normal and tangential force components:

$$
\begin{array}{r}
F_{\text {norm }}=\varepsilon_{w} \sum_{i_{1}}^{N_{x}^{1}} \sum_{i_{2}}^{N_{x}^{2}} \sum_{j_{1}}^{N_{y}^{1}} \sum_{j_{2}}^{N_{y}^{2}} \sum_{k_{1}}^{N_{z}^{1}} \sum_{k_{2}}^{N_{z}^{2}} \frac{24}{\sigma_{w}\left(r_{1}-r_{2}\right)^{2}}\left(\left(\frac{\sigma_{w}}{r_{1}-r_{2}}\right)^{7}-2\left(\frac{\sigma_{w}}{r_{1}-r_{2}}\right)^{13}\right) \\
\times\left(y_{j_{1}}^{1}-y_{j_{2}}^{2}\right) ; \\
F_{\text {tang }}^{x}=\varepsilon_{w} \sum_{i_{1}}^{N_{x}^{1}} \sum_{i_{2}}^{N_{x}^{2}} \sum_{j_{1}}^{N_{y}^{1}} \sum_{j_{2}}^{N_{y}^{2}} \sum_{k_{1}}^{N_{z}^{1}} \sum_{k_{2}}^{N_{z}^{2}} \frac{24}{\sigma_{w}\left(r_{1}-r_{2}\right)^{2}}\left(\left(\frac{\sigma_{w}}{r_{1}-r_{2}}\right)^{7}-2\left(\frac{\sigma_{w}}{r_{1}-r_{2}}\right)^{13}\right) \\
\times\left(x_{i_{1}}^{1}-x_{i_{2}}^{2}\right) ;
\end{array}
$$


Here,

$$
\begin{aligned}
& N_{x}^{1}=N_{z}^{1}=L \sqrt[3]{\frac{\rho N_{A}}{\mu_{\mathrm{H}_{2} \mathrm{O}}}} ; N_{y}^{1}=H_{\mathrm{ice}} \sqrt[3]{\frac{\rho N_{A}}{\mu_{\mathrm{H}_{2} \mathrm{O}}}} ; \\
& N_{x}^{2}=N_{z}^{2}=L \sqrt[3]{\frac{\rho N_{A}}{\mu_{\mathrm{wall}}}} ; N_{y}^{2}=H_{\text {wall }} \sqrt[3]{\frac{\rho N_{A}}{\mu_{\text {wall }}}} .
\end{aligned}
$$

From this system of equations after measurement of forces of adhesion in normal and tangential directions, one can find two LJ parameters for interaction between single atom and a water molecule. Knowing these constants provides a physical mechanism of the adhesion force (between ice and a rough nanostructured surface) which can be used in numerical simulations of ice accretion. After obtaining the values of these parameters, one can create a fragment of a rough (or nanostructured aircraft surface as well as a surface which is covered by antiicing liquid) in order to calculate a force which keeps the ice on aircraft surface. After precisely calculating the force which acts on a tiny fragment of a surface, it is easy to obtain a value of ice adhesion force which acts on a bigger fragment whose size equals the size of a mesh in numerical simulations. Numerical calculations of the ice accretion could decrease a number of expensive flight experiments as well as ground tests for ice accretion investigations.

\section{CONCLUDING REMARKS}

Macrocharacteristics of a nanoparticle are presented in terms of intermolecular interaction laws. The method is derived to determine an area in the multiparameter domain of flight conditions (velocity, temperature, cluster dimensions, etc.) in which a water nanoparticle could freeze upon the aircraft surface and to determine the laws of collision of water nanoparticles in order to define a physical mechanism of condensation. Measurements of adhesion forces between the ice and an aircraft surface allow to determine the laws of interaction between a water molecule and a surface atom. A procedure of ice adhesion simulation is proposed in order to take into account a cicumfluent body surface nanorelief and special chemical properties of the material.

\section{Additional Remarks}

If the water or ice particulate is regarded as the macroscopic one, it should follow a certain averaged trajectory, and the larger is the particulate, the more readily it impinges a circumfluent body. However, the real movement of the particulate corresponds not only to the averaged line, but also to the Brownian and turbulent 
pulsations, whose role augments with diminishing of the particulate dimension. In the limit of a single molecule, it may impinge the solid body surface due to thermal stochastic inclination from the gas flow lines. As a result, the particle-tosurface impingement probability depends on the particle size nonmonotonically but never tends to zero.

So, the present investigations had two aims: first, to take into consideration such a possibility not for a molecule, but also for a molecular cluster; and second, to derive an algorithm which could be readily used in the limit of the larger drops (in the micrometer scale) when computation techniques would become mighty enough.

As a result, a domain in the multiparameter space of the flight conditions (velocity, temperature, liquid water content, etc.) and physical properties of the water-to-surface contact (wetting angle, surface energy density, etc.) may be determined in which there is no ice accretion. Consequently, corresponding recommendations could be formulated for the industry of materials in terms of physical properties which are easily measured in laboratory conditions.

\section{ACKNOWLEDGMENTS}

These investigations were supported by the Russian Foundation for Basic Research (Grant No. 13-01-00766), and also in the network of Federal Task Program for 2009-2013 (State Contracts Nos. 14.740.11.0576 and 14.740.11.1072).

\section{REFERENCES}

1. Böttger, R. 1935. Untersuchungen über die Eignung von Scheibe und Kegel zur Messung des Eisbelagesmittel des Aerodynamishen Wielderstandes. IZWB FB. Berlin 462:12 S.

2. Bidwell, C. S. 1996. Collection efficiency and ice accretion calculations for a Boeing 737-300 inlet. AIAA Paper No. 96-5570.

3. Birch, S. 1999. Simulating aircraft icing. Aerospace Eng. 19(10):19-20.

4. Cebeci, T., and F. Kafyeke. 2003. Aircraft icing. Ann. Rev. Fluid Mech. 35:11-21.

5. Stasenko, A. L., A.I. Tolstykh, and D. A. Shirobokov. 2002. Deformable-drop dynamics near an airfoil surface in viscous air. Fluid Dyn. 37(5):825-833.

6. Kashevarov, A. V., and A. L. Stasenko. 2010. Force crystallization of drops in front of a body moving in a supercooled cloud. Math. Mod. Computer Simulations $2(5): 614-620$.

7. Artyukhin, A.S., B. V. Egorov, A. L. Chugrieev, B. V. Egorov, A. Yu. Khlopkov, A. V. Kosheev, Yu.E. Markachiov, E. A. Plekhanov, I.S. Ufimtsev, and 
E. A. Zababurin. 2004. Kinetics of ultralight neutral and electrical charged fractions of clusters formation in aerodynamic fluxes of flyuing chehicle. Khim. Fiz. 24:28-46. [In Russian.]

8. Granovsky, A. Available at: http://classic.chem.msu.su/gran/games/index.html (accessed September 1, 2014).

9. Rahman, A., and F. Stillincer. 1985. Molecular dynamics study of liquid water. J. Chem. Phys. 55(7):3336-3359.

10. Chaplin, M. 2000. A proposal for the structuring of water. Biophys. Chemist. 83(3):211-221.

11. Matsumoto, M. 2011. Nanoscale bubbles and droplets. Investigation with molecular simulations. 8th Pacific Symposium on Flow Visualization and Image Processing. Moscow: Lomonosov Moscow State University. CD.

12. http://isbu.ac.uk/water/models.html (accessed August 22, 2014).

13. Prigogine, I. R., and I. Stengers. 1984. Order out of chaos. Man's new dialogue with nature. London: Heinemann. 432 p.

14. Allen, M., and D. Tildesley. 1987. Computer simulation of liquids. London: Clarendon Press. 385 p.

15. Kaplan, I. G. 2006. Intermolecular interactions: Physical picture. Computational methods and model potentials. John Wiley \& Sons. 368 p.

16. Car, R., and M. Parrinello. 1985. Unified approach for molecular dynamics and density-functional theory. Phys. Rev. Lett. 55(22):2471-2474.

17. Verlet, R. 1967. Loup computer experiments on classical fluids. I. Thermodynamical properties of Lennard-Jones molecules. Phys. Rev. 159:98-103.

18. Tolman, R. C. 1949. Effect of droplet size on surfaces tension. J. Chem. Phys. 17:333-338.

19. Nevzorov, A. N. 2006. Internal mechanism of metastable liquid water crystallization and its effects on intracloud processes. Russ. Akad. Sci. Atmospheric Oceanic Phys. 42(6):765-772.

20. Radchenko, I. V. 1965. Molecular physics. Moscow: Nauka. 480 p. [In Russian.] 